\title{
Robust Control of Quantum Information
}

\author{
Marco A. Pravia \\ Department of Nuclear Engineering, Massachusetts Institute of Technology, Cambridge, MA 02139 and \\ Department of Computer Science, University of Puerto Rico, Rio Piedras, PR 00931 \\ Nicolas Boulant, Joseph Emerson and Evan M. Fortunato, Timothy F. Havel, David G. Cory \\ Department of Nuclear Engineering, Massachusetts Institute of Technology, Cambridge, MA 02139 \\ Amro Farid \\ Department of Mechanical Engineering, Massachusetts Institute of Technology, Cambridge, MA 02139
}

Errors in the control of quantum systems may be classified as unitary, decoherent and incoherent. Unitary errors are systematic, and result in a density matrix that differs from the desired one by a unitary operation. Decoherent errors correspond to general completely positive superoperators, and can only be corrected using methods such as quantum error correction. Incoherent errors can also be described, on average, by completely positive superoperators, but can nevertheless be corrected by the application of a locally unitary operation that "refocuses" them. They are due to reproducible spatial or temporal variations in the system's Hamiltonian, so that information on the variations is encoded in the system's spatiotemporal state and can be used to correct them. In this paper liquid-state nuclear magnetic resonance (NMR) is used to demonstrate that such refocusing effects can be built directly into the control fields, where the incoherence arises from spatial inhomogeneities in the quantizing static magnetic field as well as the radio-frequency control fields themselves. Using perturbation theory, it is further shown that the eigenvalue spectrum of the completely positive superoperator exhibits a characteristic spread that contains information on the Hamiltonians' underlying distribution.

PACS numbers: 03.67.-a; 02.30.Yy, 02.30.Zz, 82.56.Jn 


\section{INTRODUCTION}

Methods of controlling quantum systems [1, 2, 3, 4, 5] are needed to direct the course of chemical reactions [ㅁ, 7], to determine molecular structure [8], and to achieve quantum information processing [9]. The common goal is to preserve or manipulate a quantum system so that the effective evolution over a control sequence is precisely the desired process. The causes of unwanted dynamics include irreversible couplings to the environment (decoherence), static or slowly varying fluctuations in the system's Hamiltonian (incoherence), and systematic unitary errors. Here, we examine a common class of experimental imperfections characterized by a spatially or temporally "incoherent variation" in the system's Hamiltonian. In addition, we describe a method for mitigating this class of errors, and we report an experimental demonstration using liquid-state nuclear magnetic resonance (NMR) techniques [8].

Experimental limitations present an important set of challenges to achieving precise control over quantum systems. Incoherent errors, which are often present in experiments, may be distinguished from decoherent because they are in principle refocusable. In the case of a spatially distributed ensemble interacting with a field, the field amplitude and direction can vary over the ensemble so that the ensemble average dynamics becomes a convex sum of spatially distinct unitary processes. The ensemble's dynamics will in general appear non-unitary, but as long as the correlation between the spins' location and the strength/direction of the field remains unchanged the dispersion in the dynamics can be refocused, at least in principle.

Spatially incoherent errors have been a recurring topic of interest in the field of NMR, where they arise as inhomogeneities in the static and radio-frequency (RF) fields involved. The spatially incoherent evolution caused by the inhomogeneities dephases the spins in the NMR ensemble, attenuating and rotating the final state away from the desired state. Past methods of refocusing incoherent evolution have been directed mainly towards achieving rotations of specific states rather than implementing a desired unitary operation on all possible states. Examples include "composite pulses" [10, 11, 12, 13, 14], which have played an important role in creating the robust RF pulse sequences used for spin inversion, spin excitation, and decoupling. Adiabatic pulses have also been used to overcome the problem of RF inhomogeneity [15, 16. In addition, average Hamiltonian methods [2] have been applied to create pulse sequences that depend mostly on phase changes in the RF fields to control the spins, making the RF field's amplitude a less important experimental parameter [17]. Most of this work has been devoted to designing robust one-spin operations, but more recently the work has been extended, in the context of quantum information processing, to include two-spin operations [18].

Here, we extend our previously described method [19] for creating RF control gates in liquid-state NMR quantum information processing experiments [20, 21, 22, 23]. The gates use RF fields that strongly modulate a system's dynamics so as to refocus all the undesired evolutions and to achieve a specific desired unitary transformation with high precision. The method makes use of numerical optimization and complete knowledge of the system's internal Hamiltonian to find a modulation of the RF field such that the effective Hamiltonian over the period for which it is applied is precisely the desired transformation. In this paper, we extend this method so as to also use knowledge of the incoherent distribution of errors in an effort to design pulses that are robust over the entire distribution and thus yield the desired transformation with even higher fidelity.

\section{MODELING, MEASUREMENT AND ANALYSIS OF INCOHERENT PROCESSES}

In quantum information processing, the central experimental goal is to efficiently generate any desired unitary operation on a quantum system. Unitary operations are realized by manipulating externally-controlled parameters in the system's Hamiltonian. The problem of finding the appropriate external parameters, however, is an inverse problem, and analytical solutions are not available except for the simplest cases. As described below, we can utilize the more easily solved forward problem in a numerical search for a control field modulation that solves the inverse problem, at least in small Hilbert spaces. The density matrix evolution under an incoherent process appears to evolve in a larger space with a continuum of parameters, denoted by $\vec{r}$, describing the variations over the ensemble. In NMR, $\vec{r}$ would be the spatial location of the individual molecules. Every measurement is an integral over $\vec{r}$, so assuming that the local density matrix $\rho(\vec{r})$ is uncorrelated with the local unitary operation $U(\vec{r})$, the ensemble-average density matrix $\rho_{i n}=\int \rho(\vec{r}) d \vec{r}$ evolves in Hilbert space as

$$
\rho_{\text {out }}=\int U(\vec{r}) \rho_{\text {in }} U^{\dagger}(\vec{r}) d \vec{r}
$$

The variation of the operator $U(\vec{r})$ is tied to the variation of a field in the corresponding Hamiltonian. The evolution can be expressed in matrix form as a superoperator acting on Liouville space, i.e.

$$
\left|\rho_{\text {out }}\right\rangle=\int(\bar{U}(\vec{r}) \otimes U(\vec{r})) d \vec{r}\left|\rho_{\text {in }}\right\rangle
$$


where $|\rho\rangle$ is the columnized density matrix (obtained by stacking its columns on top of each other), $\bar{U}$ is the complex conjugate of $U$ and " $\otimes$ " is the Kronecker product of the matrices [8, 24]. In this form, it is clear that the input state $\left|\rho_{\text {in }}\right\rangle$ is transformed by the superoperator

$$
S=\int(\bar{U}(\vec{r}) \otimes U(\vec{r})) d \vec{r}
$$

to produce the final state $\left|\rho_{\text {out }}\right\rangle$. So although every element of the ensemble evolves unitarily, the ensemble-average density matrix undergoes non-unitary dynamics.

\section{A. Incoherent Processes in NMR Spectroscopy}

In the specific case of liquid-state NMR, the task of quantum control is to determine the RF pulse sequence that modulates the internal spin Hamiltonian of every molecule so as to generate a desired evolution. The homonuclear internal Hamiltonian for a molecule containing $N$ spin $1 / 2$ nuclei is

$$
H_{\text {int }}=\sum_{k=1}^{N}-\gamma_{k}\left(1-\sigma_{z}^{k}\right) B_{0}(\vec{r}) I_{z}^{k}+2 \pi \sum_{j>k}^{N} \sum_{k=1}^{N} J_{k j} I^{k} \cdot I^{j},
$$

where $-\gamma_{k}\left(1-\sigma_{z}^{k}\right) B_{0}(\vec{r})$ represents the chemical shift frequency of the $k$ th spin ( $\gamma$ is the gyromagnetic ratio and $\sigma_{z}$ is the shielding constant), $J_{k j}$ is the coupling constant between spins $k$ and $j$ and $I_{i}$ denotes the i axis spin angular momentum operator. The chemical shifts are functions of space because the main magnetic field is never perfectly homogeneous througout the sample. The scalar coupling constants, however, are independent of location since they depend only on molecular bonding.

The corresponding experimentally-controlled RF Hamiltonian is

$$
H_{e x t}(t)=\sum_{k=1}^{N}-\gamma_{k} f(\vec{r}) B_{R F}(t) e^{-i \phi(t) I_{z}^{k}} I_{x}^{k} e^{i \phi(t) I_{z}^{k}},
$$

where the time-dependent functions $B_{R F}(t)$ and $\phi(t)$ specify the applied RF control field, while $f(\vec{r})$ reflects the RF field strength distribution over the sample. Here, we assume that only phase changes in time are important and drop the spatial dependence of $\phi$.

Excluding decoherence, the evolution generated by the above Hamiltonians between time 0 and $\tau_{f}$ is

$$
U_{\text {gate }}(\vec{r})=T \exp \left(-i \int_{0}^{\tau_{f}}\left[H_{\text {int }}(\vec{r})+H_{\text {ext }}(\vec{r}, t)\right] d t\right)
$$

where $T$ is the Dyson time-ordering operator. The goal is to determine a function $B_{R F}(t)$ and $\phi(t)$ that results in a net evolution that at every location is close to the desired transformation. In general, this inverse problem is difficult to solve but the forward problem of evaluating $U_{\text {gate }}$ is readily solved numerically for small spin systems.

To efficiently calculate $U_{\text {gate }}(\vec{r})$, several simplifications can be made. First, the use of shim controls allows the static magnetic field homogeneity to be made less than 1 part in $10^{8}$ over the sample volume, meaning that the resonance frequencies of magnetically equivalent spins differ by the same amount. As a result, for now we will drop the spatial dependence of $B_{0}$; the major problem is the $\mathrm{RF}$ field variations in $H_{\text {ext }}$. We have shown previously that in the fully coherent case strongly modulating RF pulses with piecewise constant RF amplitudes provide an easily computed modulation sequence with sufficient control over the spins [19]. The evolution of a single pulse with constant (in time) RF power $B_{R F}$, phase $\phi$, frequency $\nu_{R F}$, and of duration $\tau$, can be solved with one frame transformation and a single diagonalization in that frame. This simplification allows the net propagator of a train of $M$ such square pulses to be written as

$$
U_{\text {gate }}=\prod_{m=1}^{M} U_{z}^{-1}\left(\nu_{R F, m}, \tau_{m}\right) \exp \left[-i H_{e f f}^{m}\left(B_{R F, m}, \nu_{R F, m}, \phi_{m}\right) \tau_{m}\right]
$$

where $U_{z}^{-1}\left(\nu_{R F, m}, \tau_{m}\right)$ executes the rotating-frame transformation of the $m$ th period and $H_{\text {eff }}$ is the effective, timeindependent Hamiltonian in the new frame of reference [8]. Using the standard simplex search algorithm, a set of parameters which match $U_{\text {gate }}$ to a desired transformation can often be obtained [19]. In the coherent case, we found 
the dynamics of these pulses to be very rich, admitting many different strongly-modulating pulses for a given ideal unitary gate. Here we extend this solution to the case of incoherent processes in the control Hamiltonian.

Radio-frequency power inhomogeneity was incorporated into the parameter search by tabulating a discrete histogram of the RF power variations. This distribution of RF power defines a Kraus operator sum [9, 24] instead of the unitary transformation of Eq. (7), namely

$$
\rho_{\text {out }}=\sum_{k} A_{k} \rho_{\text {in }} A_{k}^{\dagger}
$$

where $A_{k}=\sqrt{p_{k}} U_{k}$ and $p_{k}$ is the fraction of the ensemble that undergoes a unitary transformation $U_{k}$. The operators $U_{k}$ were evaluated using Eq. (7), and they differ only in the RF amplitudes involved. In NMR spectrometers the frequency and duration of the pulse do not vary as a function of position, and although the absolute phase does, this phase is unobservable here since the same RF coil is used for both transmission and reception.

The RF inhomogeneity of our experimental setup was measured using a spin nutation experiment in which the transverse magnetization of the spin system was measured after on-resonance pulses of increasing duration. The power spectrum of nutation frequencies (Fig. 1) is a direct measure of the distribution of RF field strengths over the sample.

The inclusion of RF inhomogeneity in the calculation of the fidelity, as outlined in section $\amalg B$, increases the required computational resources per iteration by a factor equal to the number of intervals used to define the distribution. Hence for the design of the pulses here only 9 intervals were used, and this distribution is superimposed on the experimental measurement in Fig. 1 (gray line).

\section{B. Metrics for the precision of coherent control}

Two useful metrics of coherent control are the state correlation and the gate fidelity [19]. The correlation measures the closeness of two density matrices $\rho_{\text {ideal }}$ and $\rho_{\text {out }}$ resulting from the same input state $\rho_{\text {in }}$ but evolved under different propagators, one of which is an ideal gate $U_{i d e a l}$ such that

$$
\rho_{\text {ideal }}=U_{\text {ideal }} \rho_{\text {in }} U_{\text {ideal }}^{\dagger}
$$

while the other is the simulated non-unitary transformation given by Eq. (8)

$$
\rho_{\text {out }}=\sum_{k} A_{k} \rho_{\text {in }} A_{k}^{\dagger}
$$

- The non-unitary transformation is trace-preserving and completely-positive. The correlation between the traceless parts $\hat{\rho}_{\text {ideal }}$ and $\hat{\rho}_{\text {out }}$ of the density matrices $\rho_{\text {ideal }}$ and $\rho_{\text {out }}$ is

$$
C\left(\hat{\rho}_{\text {ideal }}, \hat{\rho}_{\text {out }}\right)=\frac{\operatorname{trace}\left(\hat{\rho}_{\text {ideal }} \hat{\rho}_{\text {out }}\right)}{\sqrt{\operatorname{trace}\left(\hat{\rho}_{\text {ideal }}^{2}\right) \operatorname{trace}\left(\hat{\rho}_{\text {out }}^{2}\right)}},
$$

which varies from -1 to 1 . The correlation reports only similarities in the "direction" between two density matrices. To account for the loss of information due to non-unitary operations, an attenuation factor is inserted, leading to the attenuated correlation, which is defined by

$$
\begin{aligned}
C_{A}\left(\hat{\rho}_{\text {ideal }}, \hat{\rho}_{\text {out }}\right)= & C\left(\hat{\rho}_{\text {ideal }}, \hat{\rho}_{\text {out }}\right) \sqrt{\frac{\operatorname{trace}\left(\hat{\rho}_{\text {out }}^{2}\right)}{\operatorname{trace}\left(\hat{\rho}_{\text {in }}^{2}\right)}} \\
& =\frac{\operatorname{trace}\left(\hat{\rho}_{\text {ideal }} \hat{\rho}_{\text {out }}\right)}{\sqrt{\operatorname{trace}\left(\hat{\rho}_{\text {ideal }}^{2}\right) \operatorname{trace}\left(\hat{\rho}_{\text {in }}^{2}\right)}} .
\end{aligned}
$$

The attenuation term quantifies the amount of information lost due to a decrease in the magnitude of $\hat{\rho}_{\text {out }}$ relative to $\hat{\rho}_{\text {in }}$.

The gate fidelity $F$ is a measure of the precision of an operation $[19,25,26]$

$$
F=\overline{C_{A}\left(\hat{\rho}_{\text {ideal }}, \hat{\rho}_{\text {out }}\right)}
$$

where the overline notation $\overline{C_{A}}$ represents the average attenuated correlation over a complete basis of orthonormal Hermitian matrices $\rho_{j}$ (i.e., trace $\left.\left[\rho_{j} \rho_{k}\right]=\delta_{j k}\right)$. In the case of incoherent processes, it is more convenient to use the equivalent expression for $F$ directly in terms of $U_{\text {ideal }}$ and the Kraus operators $\left\{A_{k}\right\}$, namely

$$
F=\frac{1}{2^{2 N}} \sum_{k}\left|\operatorname{Tr}\left(U_{\text {ideal }}^{\dagger} A_{k}\right)\right|^{2}
$$

where $N$ is the number of qubits, which can be evaluated more rapidly than the form in Eq. (14). 


\section{Exploring the Achievable Fidelities}

In this subsection, we briefly revisit the coherent case. The ideal single-spin gate fidelities of the pulses previously achieved values over 0.999 in numerical simulations [19]. Two important experimental parameters place restrictions on the achievable fidelities, the main field strength $B_{0}$, which determines how well the individual spins are resolved, and the maximum allowed RF power, which limits the modulation rate. To learn how changing these parameters affects the fidelity of a gate, we evaluted the fidelity for a $\pi / 2]_{x}^{2}$ alanine pulse as a function of allowed main field strength and RF amplitude. The results are illustrated in Fig. 2

The three curves represent the results for each of three static magnetic field strengths tested. Each point represents the highest fidelity found by the pulse search method out of several attempts to find the gate at that magnetic field and RF power. The stronger static fields enabled pulses with higher fidelities because the stronger fields cause the differences among the spins' frequencies to increase, allowing more lattitude for addressability and control. The fidelities also increased monotonically with the maximum allowed RF power. At low powers, the RF control is insufficient to average out the internal Hamiltonian, resulting in low fidelities, while at high RF power, the strength of the RF dominates the internal Hamiltonian and hence allows the dynamics to be more precisely controlled.

It is important to emphasize that the maximum achievable fidelities of Fig. 22 represent the best gates achieved using the current pulse parameterization and search method and the available computational resources. These results do not preclude other methods and search strategies from finding higher fidelity gates. In addition, the analysis explores an optimistic scenario in which natural decoherence and experimental imperfections, including RF field inhomogeneity, are ignored. Nevertheless, expect these trends will also hold for the "self-refocusing" pulses developed in this paper.

\section{DESIGNING PULSES THAT COMPENSATE FOR RF INHOMOGENEITY}

The spatial incoherence of the RF amplitude, or RF inhomogeneity, in NMR is a consequence of the need for high sensitivity, which necessitates wrapping the RF coil tightly around the sample. As a result, different parts of the NMR sample feel unequal RF fields, causing a dephasing of the spins that attenuates the signal and introduces errors in the rotations of the spins. The problem can be avoided by using smaller parts of the sample (and thus less signal). An alternative, however, is to take advantage of the reproducibility of the field strength distribution so as to design gates that are insensitive to these errors.

We used the methods of section $\Pi$ to search for both compensated and uncompensated modulation sequences for 11 single qubit transformations. The calculations were performed for the three-spin system consisting of the ${ }^{13} \mathrm{C}$-nuclei in isotopically-enriched alanine. Fig. 3 summarizes the simulated fidelities for the resulting gates.

Each fidelity point on the left of Fig. 3 was calculated for a unitary gate having a single RF field strength. Each curve traces the gate fidelity as a function of the deviation from the ideal RF field strength. The plot shows that the compensated pulses are significantly less sensitive to changes in the RF modulation strength. The uncompensated pulses, however, have the overall highest fidelities when the RF amplitude is at its optimum value. This fact is confirmed in the more realistic situation where a spectrum of RF frequencies is present. The plot on the right of Fig. 3 graphs the pulse fidelities for the non-unitary transformations generated with RF distributions of varying widths. The distributions used were stretched or narrowed versions of the measured RF profile (Fig. 1), and the widths have been rescaled such that a value of 1.0 corresponds to the experimentally measured RF profile.

\section{EIGENVALUE SPECTRA OF SUPEROPERATORS}

In this section, we present a numerical study of the action of both compensated and uncompensated gates by looking at the eigenvalue spectrum of the superoperators of the gates. We will show how the eigenvalues of the superoperators can serve as a useful and convenient tool for extracting information about the imperfections in the implementations of unitary transformations. In particular, we shall describe features of the eigenvalue spectrum that allow us to characterize the distribution of incoherent processes in the superoperators, so that the reduction of these features in our simulated superoperators provides further evidence for the closer-to-unitary behavior of compensated gates as compared to uncompensated ones.

\section{A. Perturbation Analysis of the Eigenvalue Spectrum}

The eigenvalues of operators in $\mathrm{SU}\left(2^{N}\right)$ lie on a unit circle in the complex plane. Let $N$ be the number of spins and $U_{k}$ denote the unitary operator determined by the RF field in the $k$ th frequency interval of the RF amplitude profile 
(as shown in Fig. 1). The eigenvalues of the superoperator obtained by taking the Kronecker product of $U_{k}$ with its complex conjugate, $\bar{U}_{k} \otimes U_{k}$, are the products of the eigenvalues of $U_{k}$ with those of $\bar{U}_{k}$, yielding $2^{N}$ eigenvalues that are equal to unity and $2^{2 N-1}-2^{N-1}$ pairs of complex conjugate eigenvalues. As a consequence, the weighted sum of the superoperators $\bar{U}_{k} \otimes U_{k}$ forces the net superoperator $S$ to have eigenvalues that are symmetric with respect to the real axis (which is essentially a consequence of the fact that $S$ preserves Hermiticity) and inside the unit circle.

Estimates of the actual eigenvalues of $S$ will now be obtained using first-order pertubation theory. Because the pulses are not perfect even in the absence of RF field inhomogeneity, we may assume that the unperturbed eigenvalues are generically non-degenerate. The unitary operator $U_{k}$ may be written in exponential form as

$$
U_{k}=e^{-i H_{k} t}
$$

where $H_{k}$ represents the effective Hamiltonian of the evolution over the period $t$ for which the pulse is applied. Defining $H_{0}$ to be the unperturbed Hamiltonian (taken to be at the peak of the profile in Fig. (1), the eigenvalues $\phi_{j}$ and eigenkets $\left|\phi_{j}\right\rangle$ of $H_{0}$ satisfy the eigenvalue equation

$$
U_{0}\left|\phi_{j}\right\rangle=e^{-i \phi_{j} t}\left|\phi_{j}\right\rangle
$$

where $U_{0}=\exp \left(-i H_{0} t\right)$. Note that this implies $\bar{U}_{0} \overline{\left|\phi_{j}\right\rangle}=e^{i \phi_{j} t} \overline{\left|\phi_{j}\right\rangle}$, where $\overline{\left|\phi_{j}\right\rangle}$ is the complex conjugate of the ket $\left|\phi_{j}\right\rangle$. The Hamiltonian of a particular $U_{k}$ can be written as

$$
H_{k}=H_{0}+K_{k}
$$

where $K_{k}$ is the perturbation. To first order, the new eigenvalues of $H_{k}$ are

$$
\tilde{\phi}_{j, k}=\phi_{j}+\left\langle\phi_{j}\left|K_{k}\right| \phi_{j}\right\rangle
$$

The corresponding eigenvalues of $U_{k}$ are then

$$
e^{-i \tilde{\phi}_{j, k} t}=e^{-i\left(\phi_{j}+\left\langle\phi_{j}\left|K_{k}\right| \phi_{j}\right\rangle\right) t}
$$

Given that $S=\sum_{k} p_{k} \bar{U}_{k} \otimes U_{k}$, the spectral decomposition of $S$ is

$$
S=\sum_{k} p_{k}\left(\sum_{m} e^{i \tilde{\phi}_{m, k} t} \overline{\left|\phi_{m}\right\rangle\left\langle\phi_{m}\right|} \otimes \sum_{j} e^{-i \tilde{\phi}_{j, k} t}\left|\phi_{j}\right\rangle\left\langle\phi_{j}\right|\right)
$$

and the eigenvalues of $S$ are then given approximately by

$$
\begin{aligned}
\lambda_{j m} & =\sum_{k} p_{k} e^{-i \tilde{\phi}_{j, k} t} e^{i \tilde{\phi}_{m, k} t} \\
& =\sum_{k} p_{k} e^{-i\left(\phi_{j}-\phi_{m}\right) t-i\left(\left\langle\phi_{j}\left|K_{k}\right| \phi_{j}\right\rangle-\overline{\left.\left\langle\phi_{m}\left|K_{k}\right| \phi_{m}\right\rangle\right) t}\right.} \\
& =e^{-i\left(\phi_{j}-\phi_{m}\right) t} \sum_{k} p_{k} e^{-i\left(\left\langle\phi_{j}\left|K_{k}\right| \phi_{j}\right\rangle-\left\langle\phi_{m}\left|K_{k}\right| \phi_{m}\right\rangle\right) t}
\end{aligned}
$$

where in the last line we have used the fact that the diagonal elements of any Hermitian operator are real.

\section{B. Eigenvalue Spectra of Uncompensated Pulses}

We now use the result of the previous section, Eq. (22), to calculate the first-order eigenvalues of an uncompensated $\frac{\pi}{2}$ pulse that rotates the alanine carbon spins 1 and 2 about the $x$-axis. Fig. 4 compares the numerically exact eigenvalues with the results from the approximation. The dots are the eigenvalues corresponding to the full simulation of the gate under the influence of the experimental RF inhomogeneity profile. The crosses are the eigenvalues computed by first-order perturbation theory. To calculate $\lambda_{j m}$, we first determined $K_{k}$ using

$$
\begin{aligned}
K_{k} & =i \times\left(\log \left(U_{k}\right)-\log \left(U_{0}\right)\right) / t \\
& =H_{k}-H_{0}
\end{aligned}
$$

where $\log$ is a logarithm of the matrix obtained from the principal branch and then used Eq. (22). 
Fig. 4 also shows an interesting relationship between the phase shift and the attenuation of the eigenvalues, i.e. the larger the phase shift, the larger the attenuation. To get more physical insight of the pattern emerging in Fig. 4 we now assume that $K_{k}$ can be written as $K_{k}=\left(\omega_{k}-\omega_{0}\right) K$. Note that we have dropped the dependence of $K$ with respect to $k$ (i.e. the "direction" of the effective Hamiltonian is constant across the sample, which would be expected for a strong RF pulse). This would be in fact an exact result for a one spin system on resonance. The eigenvalue expression then becomes:

$$
\begin{aligned}
\lambda_{j m} & =e^{-i\left(\phi_{j}-\phi_{m}\right) t} \sum_{k} p_{k} e^{-i\left(\left\langle\phi_{j}\left|K_{k}\right| \phi_{j}\right\rangle-\left\langle\phi_{m}\left|K_{k}\right| \phi_{m}\right\rangle\right) t} \\
& =e^{-i\left(\phi_{j}-\phi_{m}\right) t} \sum_{k} p_{k} e^{-i \psi_{j m}\left(\omega_{k}-\omega_{0}\right) t}
\end{aligned}
$$

thereby yielding in the continuous limit

$$
\begin{aligned}
\lambda_{j m} & =e^{-i\left(\phi_{j}-\phi_{m}\right) t} \int p\left(\omega_{k}\right) e^{-i \psi_{j m}\left(\omega_{k}-\omega_{0}\right) t} d \omega_{k} \\
& =e^{-i\left(\phi_{j}-\phi_{m}\right) t} \int p(\Delta \omega) e^{-i \psi_{j m} \Delta \omega t} d \Delta \omega
\end{aligned}
$$

where $\Delta \omega=\omega_{k}-\omega_{0}$ and where $\psi_{j m}$ is an unknown real quantity. We see in this case that to first order the eigenvalue $\lambda_{j m}$ is just the unperturbed eigenvalue $e^{-i\left(\phi_{j}-\phi_{m}\right) t}$ times the Fourier transform of the RF distribution profile evaluated at $\psi_{j m}$. This result demonstrates that the probability distribution profile of an incoherent process could be determined in principle from the eigenvalue structures of an experimental superoperator and a unitary-desired one. Knowing the form of $K$ would indeed allow one to build the correspondence between $\lambda_{j m}$ and $\psi_{j m}$, and then to determine $p(\Delta \omega)$ by performing an inverse Fourier transform. This result holds of course when the perturbation is in the first order regime and when the unperturbed eigenvalues are non-degenerate.

To gain further understanding about the estimation of the eigenvalues of $S, K_{k}$ can be expanded in the basis of products of Pauli spin matrices and the matrix elements contributing to the perturbation theory analysis can be determined. As an example, let us consider a one-spin system. In this case, $K_{k}$ can be expanded in terms of $\sigma_{x}, \sigma_{y}$ and $\sigma_{z}$. If we take $H_{0} t=\frac{\pi}{4} \sigma_{x}$, then we see that any operator in the expansion of $K_{k}$ that anticommutes with $\sigma_{x}$ results in zero diagonal matrix elements in the eigenbasis of $\sigma_{x}$, because

$$
\begin{aligned}
A \sigma_{x}+\sigma_{x} A=0 & \Rightarrow\left\langle \pm\left|A \sigma_{x}+\sigma_{x} A\right| \pm\right\rangle=0 \\
& \Rightarrow \pm 2\langle \pm|A| \pm\rangle=0
\end{aligned}
$$

where $| \pm\rangle$ denote the eigenstates of $\sigma_{x}$, and $A$ is an operator in the decomposition of $K_{k}$ that anticommutes with $\sigma_{x}$. In this simple example, only the component along $H_{0}$ in $K_{k}$ yields a non-zero contribution in the eigenvalue calculation given above ( $I$ is not present either). In our three-spin system, if $H_{0} t=\frac{\pi}{4} \sigma_{x}^{1}$, it follows that out of the 64 possible operators in the expansion of $K_{k}$ only $\sigma_{x}^{1}, \sigma_{i}^{2}, \sigma_{j}^{3}, \sigma_{i}^{2} \sigma_{j}^{3}, \sigma_{x}^{1} \sigma_{i}^{2}, \sigma_{x}^{1} \sigma_{j}^{3}$ and $\sigma_{x}^{1} \sigma_{i}^{2} \sigma_{j}^{3}$ will give a nonzero contribution, where $i, j=x, y$ or $z$. In general, the number of operators that yield a non-zero contribution for a $2^{n}$ by $2^{n}$ unitary operator $U$ is $2^{n}-1$, i.e. the number of diagonal elements minus 1 (the identity part). This quick analysis reveals to some extent the slight discrepancy we have between the first order perturbation theory results and the fully simulated ones. Due to the nature of our physical system, the first order perturbation approach takes into account only a small number of elements in the decomposition of $K_{k}$. However, because it captures the general distribution of the eigenvalue spectrum, we can conclude that a significant amount of the perturbation is contained in these few operators.

\section{Symmetric Inhomogeneity Profile}

To test further our analysis and the validity of our assumptions we calculated the superoperators where a symmetric inhomogeneity profile is present. We recall the previous formula

$$
\begin{aligned}
\lambda_{j m} & =e^{-i\left(\phi_{j}-\phi_{m}\right) t} \sum_{k} p_{k} e^{-i\left(\left\langle\phi_{j}\left|K_{k}\right| \phi_{j}\right\rangle-\left\langle\phi_{m}\left|K_{k}\right| \phi_{m}\right\rangle\right) t} \\
& =e^{-i\left(\phi_{j}-\phi_{m}\right) t} \sum_{k} p\left(\Delta \omega_{k}\right) e^{-i V_{j m}\left(\Delta \omega_{k}\right) t}
\end{aligned}
$$


where $V_{j m}\left(\Delta \omega_{k}\right)=\left(\left\langle\phi_{j}\left|K_{k}\right| \phi_{j}\right\rangle-\left\langle\phi_{m}\left|K_{k}\right| \phi_{m}\right\rangle\right)$ and $\Delta \omega_{k}=\omega_{k}-\omega_{0}$. Now because $p\left(\Delta \omega_{k}\right)$ is symmetric with respect to 0 , one can see that the additional assumption of $V_{j m}\left(-\Delta \omega_{k}\right)=-V_{j m}\left(\Delta \omega_{k}\right)$ (as we did in the previous subsection by setting $K_{k}=\Delta \omega_{k} K$ ) leads to the result that $\lambda_{j m}=e^{-i\left(\phi_{j}-\phi_{m}\right) t} A_{j m}$ where $A_{j m}$ is a real number smaller than 1 . In other words, in the case of a symmetric inhomogeneity profile, and provided the above assumption is valid, the eigenvalues simply get attenuated, and are not phase shifted. As one can see in Fig. 5 for a symmetric profile, the eigenvalues have practically the same phase as when there is no RF inhomogeneity and are simply attenuated by the real factor $A_{j m}$. This provides further evidence that the new eigenvalues are correlated with the Fourier transform of the inhomogeneity profile, since the Fourier transform of a symmetric real function is real.

\section{Eigenvalue Spectra of the Compensated Pulses}

As described above, pulses that are compensated for RF inhomogeneity result in an overall operation closer to being unitary than for uncompensated pulses. In Fig. [6] we compare the eigenvalue spectrum of the superoperators corresponding to a compensated and uncompensated $\frac{\pi}{2}$ pulse about the $x$ axis on the alanine spins 1 and 2 .

In general, a unitary superoperator in the Zeeman basis 24 which is trace-preserving and completely positive must correspond to a unitary process in Hilbert space, and can be written as $\bar{U} \otimes U$ (Boulant et al., in preparation). Since the superoperator of an incoherent process can be written as a trace-preserving Kraus operator sum, it must be completely positive and hence is physically reasonable. Thus the fact that the eigenvalues of the superoperators that were simulated for the compensated pulses basically lie on the unit circle provides further evidence for them being very nearly unitary.

\section{EXPERIMENTAL RESULTS}

The experimental tests were carried out on the three carbons of ${ }^{13} \mathrm{C}$-labeled alanine using a $300 \mathrm{MHz}$ Bruker Avance spectrometer (see Ref. [19] for the internal Hamiltonian's parameters). The experiments tested compensated and uncompensated versions of seven different spin-rotation pulses, which were then used to selectively evolve the two scalar couplings $J_{12}$ and $J_{23}$ while refocusing the other couplings [32]. These pulses were applied to the three input states

$$
\rho_{i n}=I_{j}^{1}+I_{j}^{2}+I_{j}^{3},
$$

where $j=x, y, z$. The input states were created using compensated or uncompensated pulses, depending on the type of gate being tested. For each gate tested, the average over the three input states was calculated and are shown in Tables【and Table【shows the results for the input states, the states obtained by selective coupling, and an average over all the single-pulse experiments, while Table $\amalg$ shows the specific results for each single-pulse experiment.

The input and output density matrices were measured using state tomography and were used in Eqs. (11) and (12) to evaluate the correlation $C$, attenuation $A$, and attenuated correlation $C_{A}\left(C_{A}=C \cdot A\right)$. In all cases, the result of state tomography on the thermal state $I_{z}^{1}+I_{z}^{2}+I_{z}^{3}$ was used as the reference for the attenuation ( $\rho_{i n}$ in Eq. (12)). State tomography [27] employs read-out pulses to rotate unobservable elements of the density matrix into observable single-spin transitions. For the three-spin alanine system used here, eight repetitions of the experiment, each with a different readout pulse, were used to reconstruct the density matrix [33].

To determine the actual experimental gate performed, however, one requires knowledge about the effect of the gate on a complete set of input states so that the full superoperator can be determined, a procedure called quantum process tomography [28, 29, 30]. To carry out process tomography for a single alanine gate requires state tomography of the input and output density matrices for a set of 64 linearly independent inputs. Thus full process tomography for one gate would involve $2 \times 64 \times 8=1024$ separate experiments. While this is certainly possible, it is quite laborious. For this reason we limited ourselves to performing state tomography on just the three input states and the corresponding output states described above. The results do not fully characterize the experimental transformations, but they provide a reasonable estimate of the quality of the gates.

The most notable difference between the results for compensated and uncompensated gates is in the severe attenuation caused by RF inhomogeneity on the uncompensated gates. The average attenuation value $A_{\text {comp }}$. for the spin rotations is nearly four times closer to unity than the corresponding value for normal pulses (i.e. $\left(1-A_{\text {uncomp. }}\right) /(1-$ $\left.\left.A_{\text {comp. }}\right)=3.92\right)$. In addition, $A_{\text {comp }}$. for the $J_{23}$ coupling is more than 10 times closer to unity than $A_{\text {uncomp }}$. although $A_{\text {comp. }}$. for $J_{12}$ is only slightly higher than $A_{\text {uncomp. }}$. The correlations for the compensated rotation gates averaged 0.991 , slightly but consistently below the average of 0.995 for the uncompensated gates. The attenuated correlations for the compensated gates, however, were higher than the corresponding values for normal pulses, and the difference 
was caused by the sharp differences in the attenuations. The results confirm that incorporating RF inhomogeneity into the pulse design can yield more robust gates and narrows the gap between the experimental implementation and the simulation results.

\section{CONCLUSIONS}

In this report, we explored the capabilities of numerically-determined strongly-modulating pulses to achieve high gate fidelities in the presence of a common variety of experimental imperfections. In particular, we showed that robustness against incoherent errors such as RF field inhomogeneity can be obtained when knowledge of these errors is incorporated into the pulse design process. The basic ideas used in this paper, i.e. strong modulation, numerical pulse-design procedures, and the incorporation of incoherent errors in these procedures, are likely to find broad applicability in the development of quantum information processing devices based in many diverse physical systems.

\section{ACKNOWLEDGEMENTS}

This work was supported by ARO, DARPA and NSF. Correspondence and requests for materials should be addressed to D. G. Cory (e-mail: dcory@mit.edu). We thank Zhiying Chen, Seth Lloyd, and Marcos Saraceno for valuable discussions.

[1] I. Rabi, S. Millman, P. Kusch, and J. Zacharias, Phys. Rev. 55, 526 (1939).

[2] U. Haeberlen and J. Waugh, Phys. Rev. 175, 453 (1968).

[3] A. Haught, Ann. Rev. Phys. \& Chem. 19, 343 (1968).

[4] W. Warren, H. Rabitz, and M. Dahleh, Science 259, 1581 (1993).

[5] W. Warren, Science 242, 878 (1988).

[6] R. J. Gordon and S. A. Rice, Annu. Rev. Phys. Chem. 48, 601 (1997).

[7] S. Rice and S. Shah, Phys. Chem. Chem. Phys. 4, 1683 (2002).

[8] R. Ernst, G. Bodenhausen, and A. Wokaun, Principles of Nuclear Magnetic Resonance in One and Two Dimensions (Oxford University Press, Oxford, 1994).

[9] M. Nielsen and I. Chuang, Quantum Computation and Quantum Information (Cambridge University Press, Cambridge, UK, 2000).

[10] M. Levitt and R. Freeman, J. Magn. Reson. 33, 473 (1979).

[11] M. Levitt, J. Magn. Reson. 48, 234 (1982).

[12] R. Tycko, Phys. Rev. Lett. 51, 775 (1983).

[13] A. Shaka and R. Freeman, J. Magn. Reson, 55, 487 (1983).

[14] M. Levitt, Prog. Nucl. Magn. Reson. Spect. 18, 61 (1986).

[15] J. Baum, R. Tycko, and A. Pines, Phys. Rev. A 32, 3435 (1985).

[16] M. S. Silver, R. I. Joseph, and D. I. Hoult, Phys. Rev. A 31, 2753 (1985).

[17] D. Cory, J. Magn. Reson 103, 23 (1993).

[18] H. Cummins, G. Llewellyn, and J. Jones, Phys. Rev. A 67, 042308 (2003).

[19] E. Fortunato, M. Pravia, N. Boulant, G. Teklemariam, T. Havel, and D. Cory, J. Chem. Phys. 116, 7599 (2002).

[20] D. Cory, A. Fahmy, and T. Havel, Proc. Natl. Acad. Sci. 94, 1634 (1997).

[21] N. Gershenfeld and I. Chuang, Science 275, 350 (1997).

[22] J. Jones, Prog. in NMR Spect. 38, 325 (2001).

[23] D. Cory, R. Laflamme, E. Knill, L. Viola, T. Havel, N. Boulant, G. Boutis, E. Fortunato, S. L. S, R. M. R, et al., Prog. Phys. 48, 875 (2000).

[24] T. Havel, J. Math. Phys. 44, 534 (2003).

[25] B. Schumacher, Phys. Rev. A 54, 2614 (1996).

[26] E. Fortunato, L. Viola, J. Hodges, G. Teklemariam, and D. Cory, New J. Phys. 4, 5.1 (2002).

[27] I. Chuang, N. Gershenfeld, M. Kubinec, and D. Leung, Proc. R. Soc. Lond. A 454, 447 (1998).

[28] I. Chuang and M. Nielsen, J. Mod. Opt. 44, 2455 (1997).

[29] A. Childs, I. Chuang, and D. Leung, Phys. Rev. A 64, 012314/1 (2001).

[30] N. Boulant, T. F. Havel, M. A. Pravia, and D. G. Cory, Phys. Rev. A 67, 042322 (2003).

[31] M. Pravia, Ph.D. thesis, Massachusetts Institute of Technology (2002).

[32] The waveforms of all the pulses were corrected using a RF feedback procedure, reducing distortions caused by amplifier nonlinearities in the experimental setup [31]. 
[33] The eight readout transformations used for the density matrix reconstruction were: identity, $\left.\left.\left.\pi / 2]_{y}^{1}, \pi / 2\right]_{y}^{12}, \pi / 2\right]_{x}^{23}, \pi / 2\right]_{y}^{3}$, $\left.\left.\pi / 2]_{x}^{3}, \pi / 2\right]_{x}^{1,2,3}, \pi / 2\right]_{y}^{1,2,3}$. All of the density matrices dicussed in this section were acquired using the eight readouts, with the exception of the input states, which used only the last seven. The last seven readouts alone are also sufficient, but we included the identity because of its particularly simple implementation. 


\section{TABLES AND FIGURES}

\begin{tabular}{|c|c|c|c|c|}
\hline $\begin{array}{l}\text { Control } \\
\text { Metrics }\end{array}$ & $\begin{array}{l}\text { Input } \\
\text { State }\end{array}$ & $\begin{array}{l}\text { Average over Seven } \\
\text { Spin-Rotation Gates }\end{array}$ & $\begin{array}{l}\text { Selective } \\
J_{12} \text { Gate }\end{array}$ & $\begin{array}{l}\text { Selective } \\
J_{23} \text { Gate }\end{array}$ \\
\hline$\left\langle C^{c o m p \cdot}\right\rangle$ & 0.993 & 0.991 & 0.986 & 0.990 \\
\hline$\left\langle C^{\text {uncomp. }}\right\rangle$ & 0.995 & 0.995 & 0.975 & 0.988 \\
\hline$\left\langle A^{\text {comp. }}\right\rangle$ & 0.998 & 0.987 & 0.954 & 0.994 \\
\hline$\left\langle A^{\text {uncomp. }}\right\rangle$ & 0.970 & 0.949 & 0.951 & 0.930 \\
\hline$\left\langle C_{A}^{\text {comp. }}\right\rangle$ & 0.991 & 0.979 & 0.941 & 0.984 \\
\hline$\left\langle C_{A}^{\text {uncomp. }}\right\rangle$ & 0.965 & 0.944 & 0.927 & 0.919 \\
\hline
\end{tabular}

TABLE I: Summary of experimental results. The metrics $C, A$, and $C_{A}$, refer to the correlation, attenuation, and attenuated correlation $\left(C_{A}=C \cdot A\right)$. The superscripts specify whether the pulses employed were compensated or uncompensated for RF inhomogeneity, while the angle brackets denote that the reported quantities are means over the three input states tested for each transformation. In the case of the spin-rotation values, the quantity reported is the average of all the spin-rotation results.

\begin{tabular}{cccccccc} 
Metrics & $\pi / 2]_{x}^{1}$ & $\pi / 2]_{x}^{3}$ & $\pi / 2]_{x}^{12}$ & $\pi / 2]_{x}^{23}$ & $\pi / 2]_{x}^{123}$ & $\pi]_{x}^{12}$ & $\pi]_{x}^{23}$ \\
\hline$\left\langle C^{\text {comp. }}\right\rangle$ & 0.991 & 0.992 & 0.991 & 0.986 & 0.994 & 0.993 & 0.992 \\
$\left\langle C^{\text {uncomp. }}\right\rangle$ & 0.996 & 0.994 & 0.996 & 0.994 & 0.996 & 0.995 & 0.995 \\
\hline$\left\langle A^{\text {comp. }}\right\rangle$ & 0.990 & 0.989 & 0.987 & 0.988 & 0.986 & 0.984 & 0.986 \\
$\left\langle A^{\text {uncomp. }}\right\rangle$ & 0.954 & 0.953 & 0.948 & 0.951 & 0.942 & 0.952 & 0.941 \\
\hline$\left\langle C_{A}^{\text {comp. }}\right\rangle$ & 0.981 & 0.981 & 0.978 & 0.974 & 0.980 & 0.977 & 0.979 \\
$\left\langle C_{A}^{\text {uncomp. }}\right\rangle$ & 0.950 & 0.948 & 0.944 & 0.945 & 0.938 & 0.947 & 0.936 \\
\hline
\end{tabular}

TABLE II: Experimental results of spin-rotation gates. The metrics $C, A$, and $C_{A}$, refer to the correlation, attenuation, and attenuated correlation $\left(C_{A}=C \cdot A\right)$. The superscripts specify whether the pulses employed were compensated or uncompensated for RF inhomogeneity, while the angle brackets denote that the reported quantities are means over the three input states tested for each transformation. The spin-rotation pulses tested were $\pi / 2$ and $\pi$ rotations of the carbon spins denoted in the superscript. 


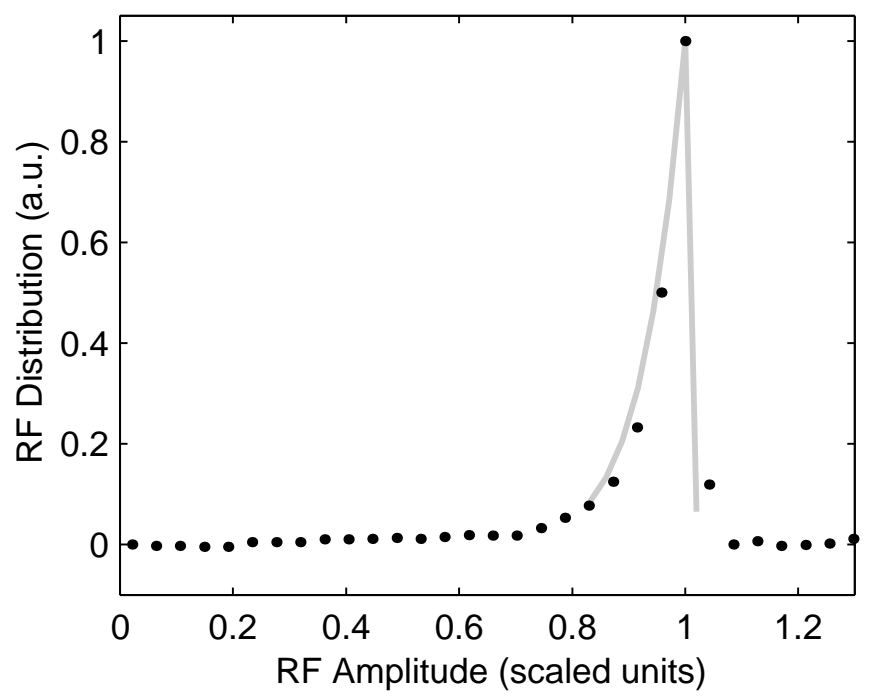

FIG. 1: Radio-frequency inhomogeneity profile. The RF inhomogeneity in the carbon channel was measured using a spin nutation experiment. The resulting decaying signal was Fourier transformed to distill the various RF nutation frequencies present in the sample. The dotted line is the plot of the Fourier transformation, and it is the measured RF inhomogeneity profile. The solid gray line is the profile that was used to design pulses compensated for RF inhomogeneity, and it was extracted from the measured profile.

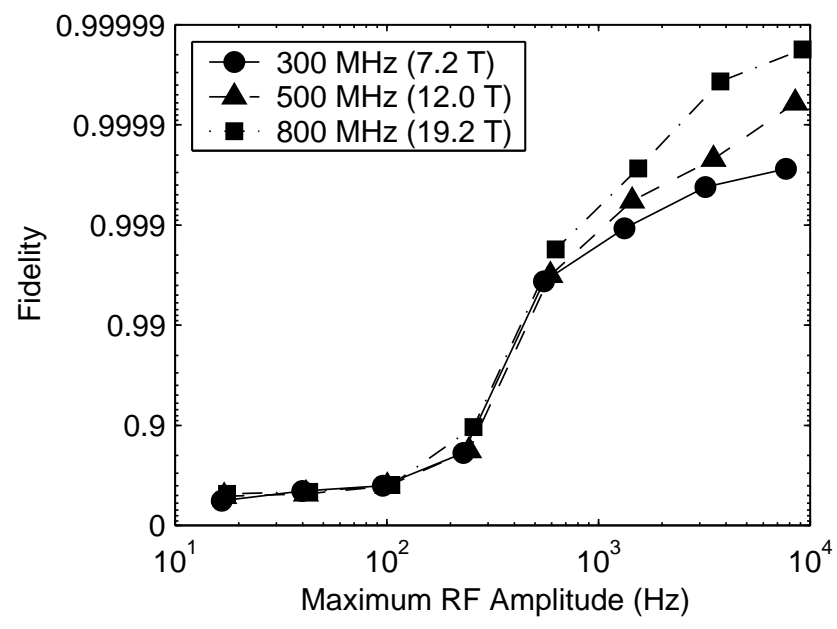

FIG. 2: Numerical exploration of achievable fidelities. The plot shows the maximum fidelities found for a $\pi / 2]_{x}^{2}$ alanine pulse as allowed RF amplitude and magnetic field strength $B_{0}$ were varied. The three lines correspond to the magnetic field strengths explored, as denoted by the legend. 

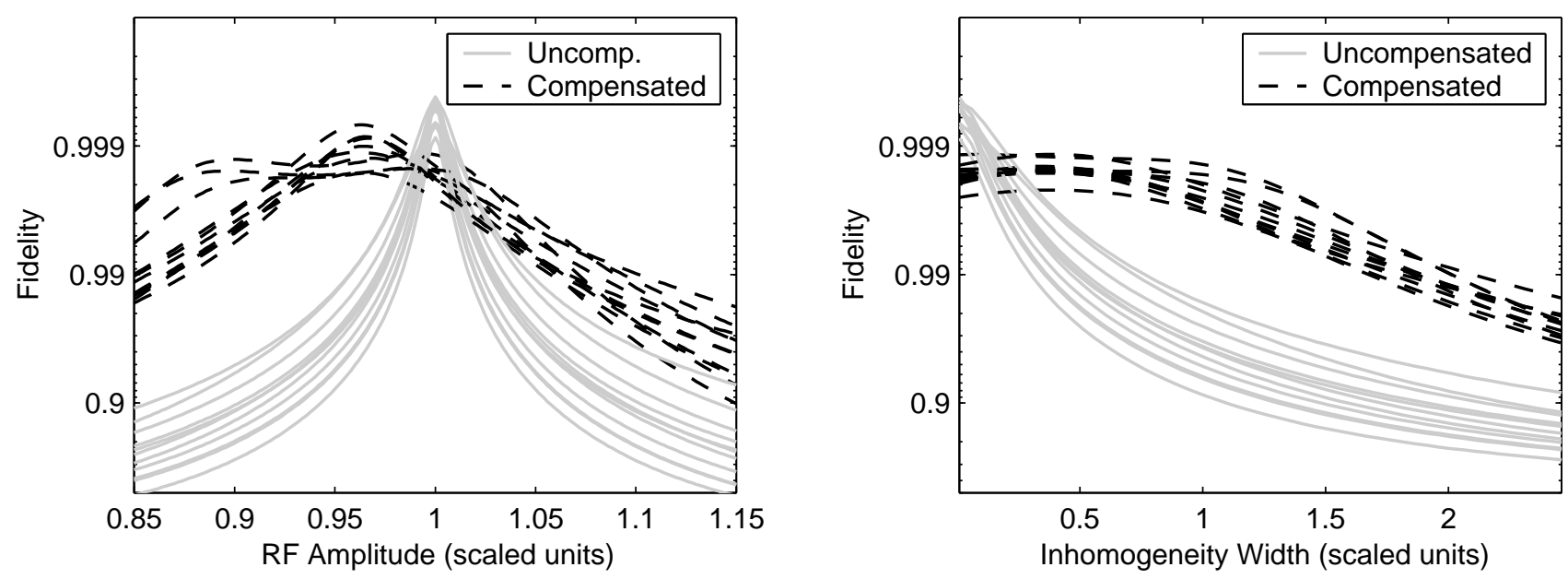

FIG. 3: Simulations of compensated and uncompensated pulses as a function of radio-frequency strengths and distribution widths. The dashed lines correspond to compensated pulses, while the solid gray lines denote uncompensated pulses. The left plot shows how the compensated pulses maintain high fidelities even when the RF strength is scaled from the ideal value. The plot on the right simulates the same pulses as a function of the scaled width of the RF inhomogeneity profile. These results demonstrate the improved fidelity of the compensated pulses for all but the narrowest RF distributions. At the small widths, the RF profile would no longer be inhomogeneous, eliminating the need for the compensated gates.
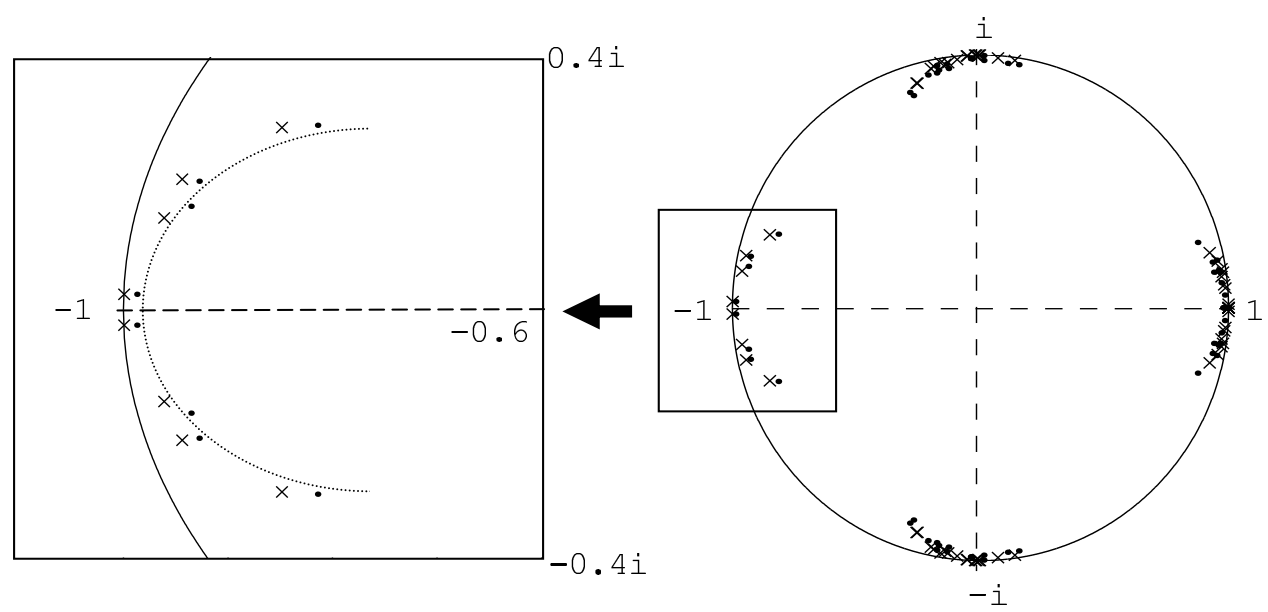

FIG. 4: Eigenvalue spectrum of the simulated superoperator for a test gate and the radio-frequency inhomogeneity profile shown in Fig. 11 The dots are the exact eigenvalues of $S$ while the crosses are the ones obtained by using the first order perturbation analysis. The zoom box shows some of the detail in the left-hand side of the plot. The additional trend line drawn in the zoomed plot emphasizes the fact that the larger the phase shift is, the larger the attenuation is. 


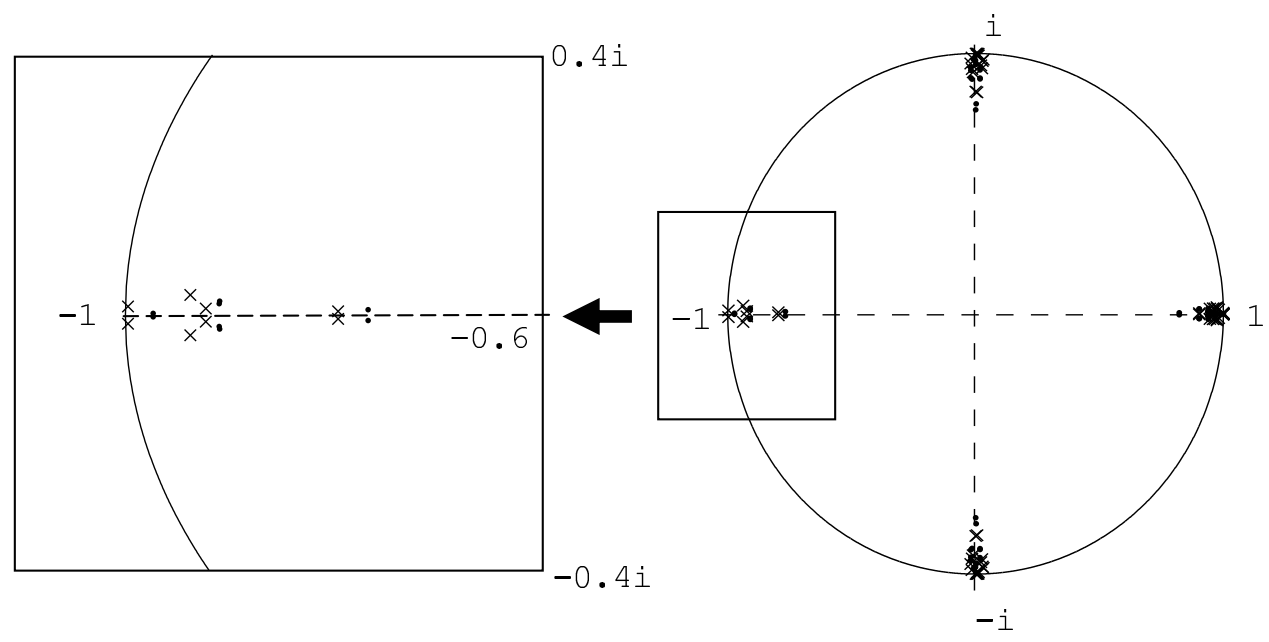

FIG. 5: Eigenvalue spectrum of the simulated superoperator for the symmetric radio-frequency inhomogeneity profile. The dots are the unperturbed eigenvalues and the crosses are the ones computed by using first order perturbation theory. The symmetry in the distribution mainly results in some attenuation of the eigenvalues with no phase shift. The zoom box allows more detail to be seen for the eigenvalues close to $-i$.

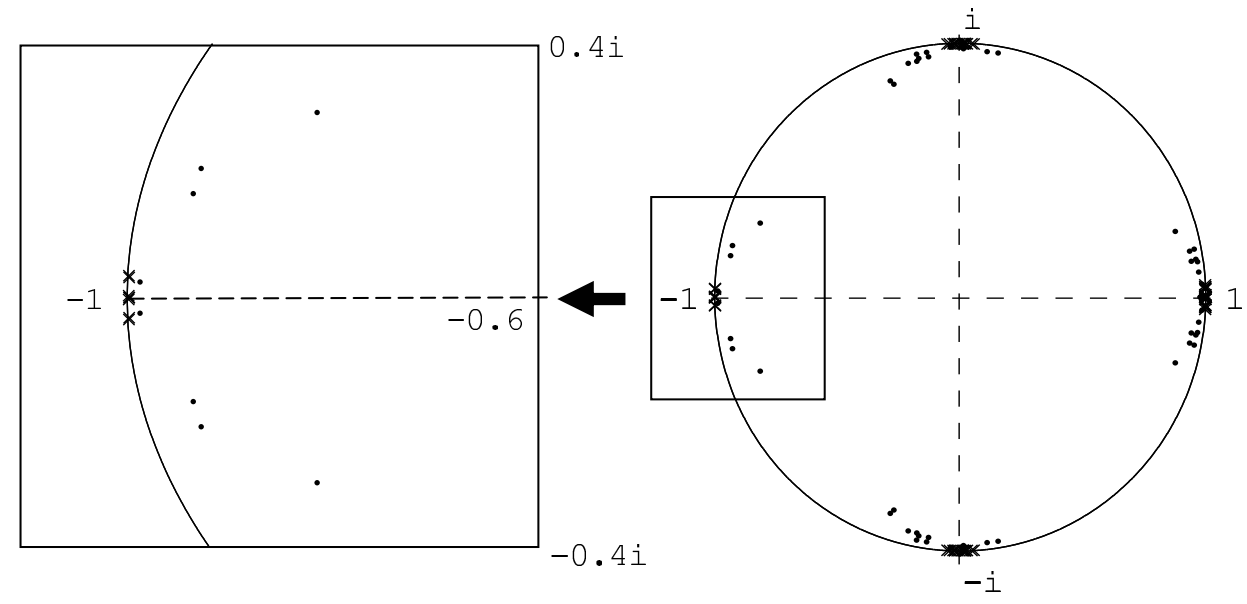

FIG. 6: Eigenvalue spectrum of the simulated superoperators corresponding to a compensated and uncompensated pulse. The dots correspond to the uncompensated gate, while the crosses correspond to the compensated one. Note that the crosses basically lie on the unit circle while the dots are spread inside, confirming the closer-to-unitary behavior of the compensated gates. 